\title{
Universal Scaling Behavior of Clustering Coefficient Induced by Deactivation Mechanism
}

\author{
Liang Tian, Chen-Ping Zhu $*$ Da-Ning Shi $\dagger$ and Zhi-Ming Gu \\ College of Science, Nanjing University of Aeronautics and Astronautics, Nanjing, 210016, PR China \\ Tao Zhou \\ Nonlinear Science Center and Department of Modern Physics, \\ University of Science and Technology of China, Hefei, Anhui, 230026, PR China
}

(Dated: August 19, 2018)

\begin{abstract}
We propose a model of network growth that generalizes the deactivation model previously suggested for complex networks. Several topological features of this generalized model, such as the degree distribution and clustering coefficient, have been investigated analytically and by simulations. A scaling behavior of clustering coefficient $C \sim 1 / M$ is theoretically obtained, where $M$ refers to the number of active nodes in the network. We discuss the relationship between the recently observed numerical behavior of clustering coefficient in the coauthor and paper citation networks and our theoretical result. It shows that both of them are induced by deactivation mechanism. By introducing a perturbation, the generated network undergoes a transition from largeto small-world, meanwhile the scaling behavior of $C$ is conserved. It indicates that $C \sim 1 / M$ is a universal scaling behavior induced by deactivation mechanism.
\end{abstract}

PACS numbers: 89.75.Hc, 87.23.Ge, 89.65.-s, 89.75.Fb

\section{INTRODUCTION}

Many social, biological, and communication systems can be properly described as complex networks with nodes representing individuals or organizations and links mimicking the interactions among them[1-3]. Examples are numerous: these include the Internet[4,5], the World Wide Web[6,7], biological networks[8,9], food webs[10], social networks[11], etc. Recent empirical studies indicate that the networks in various fields exhibit some common topological characteristics: a small average distance as random networks, large clustering coefficient as regular networks (small-world property)[12] and a power-law degree distribution (scale-free property)[13]. The ubiquity of complex networks has inspired tremendous investigations on them. Among these flourishing researches, the effect of aging is of particular interest[14-18], since it is a universal mechanism in reality. For instance, in the movie actor collaboration network, the more famous an actor is, the more chances he will have to act in new movies. But, no matter how famous he may be, every star will become gradually inactive as time goes on. This aging effect can greatly influence the evolution of networks and results in peculiar network structural property[14,15].

Recently, Börner et al. introduced a general process model that simultaneously grows coauthor and paper citation networks[19], in which the core assumption is that the twin networks of scientific researchers and academic articles mutually support one another. In their model, each of the authors and papers is assigned a topic, and

*Electronic address: chenpingzhu@yahoo.com.cn

${ }^{\dagger}$ Electronic address: shi@nuaa.edu.cn authors read, cite, produce papers or coauthor with others only in their own topic area. Interestingly, they found that the clustering coefficient $C$ of the simulated paper citation network is linearly correlated with the number of topics. We note that the main underlying dynamic rule governing the evolution of the network is aging. For example, due to the lifespan of human, once authors are older than a specified age, they will be set deactivated, and do not produce papers or coauthor with others any longer. Furthermore, papers cease to receive links when their contents are outdated. Therefore, these considerations motivate us to theoretically investigate the effect of aging on the clustering coefficient of the network. In the present paper, we concentrate on this ingredient of self-organization of the coauthor and paper citation networks and propose a simple generalized model, in which the main dynamic is deactivation mechanism. We will demonstrate that the behavior of clustering coefficient $C$ in the coauthor and paper citation networks is universal in networks generated by deactivation mechanism.

This paper is organized as follows. In section II, the model is introduced. In section III, we give both the numerical and analytic results about the effect of deactivation mechanism on network structure, including degree distribution (Sec. III A) and clustering coefficient (Sec. III B). An interesting scaling behavior of $C$ is obtained. In section IV, a structural perturbation is introduced. We show that the perturbation leads to a structural transition from large- to small-world (Sec. IV A), while the scaling behavior of $C$ is conserved (Sec. IV B). Finally in section $\mathrm{V}$, we discuss the relationship between our result and the behavior of clustering coefficient in the coauthor and paper citation networks and give a summary . 


\section{THE MODEL}

In the present model, each node can be in two different states: active or inactive[18,20,21]. The evolution process starts with a one-dimensional lattice consisting of $M$ active nodes with periodic boundary condition and coordination number $2 z[22]$. Then, in each time step

(1) Add a new node into the network, and connect it to $m$ nodes randomly chosen from the $M$ active ones.

(2) Activate the new node.

(3) Deactivate one of the active nodes. The probability that the node $i$ is deactivated is given by

$$
\pi\left(k_{i}\right)=\frac{\alpha}{k_{i}}
$$

where the normalization factor is defined as $\alpha=$ $\left(\sum_{j \in \mathcal{A}} 1 / k_{j}\right)^{-1}$. The summation runs over the set $\mathcal{A}$ of the currently $M+1$ active nodes.

It is worthwhile to note that, when $M=m$ and $z=\left[\frac{M}{2}\right]$, the present model reduces to the famous deactivation model introduced by Klemm and Eguíluz (KE model)[18]. For convenience, we call this generalized deactivation model GKE model.

\section{STRUCTURAL PROPERTIES}

\section{A. Degree distribution}

By using the continuous approximation similar to that used in Ref. [18], the degree distribution $P(k)$ can be obtained analytically for GKE model. Let us first derive the degree distribution $p^{(t)}(k)$ of the active nodes at time $t$. It evolves according to the following master equation:

$$
\begin{aligned}
p^{(t+1)}(k+1) & =p^{(t)}(k) \frac{m}{M}[1-\pi(k)] \\
& +p^{(t)}(k+1)\left[1-\frac{m}{M}\right][1-\pi(k+1)]
\end{aligned}
$$

On the right side of Eq. (2), the first term accounts for the process in which an active node with degree $k$ at time $t$ is connected to the new node and not deactivated in the next time step; The second term indicates the process that an active node with degree $k+1$ at time $t$ is not connected to the new node and still active in the next time step.

We investigate the behavior of $\alpha$ in time evolution. Fig. 1 shows the dependence of the normalization factor $\alpha$ on time $t$. We find that $\alpha$ approaches a stable value with certain fluctuations as soon as the evolution of the network starts. We assume that the fluctuations of the normalization factor $\alpha$ are small enough, i. e., it can be treated as a constant. Then, the stationary case $p^{(t+1)}(k)=p^{(t)}(k)$ of Eq. (2) yields

$$
p(k+1)-p(k)=\frac{-\alpha-(\gamma-1) k}{k(k+\gamma-\alpha)} p(k),
$$

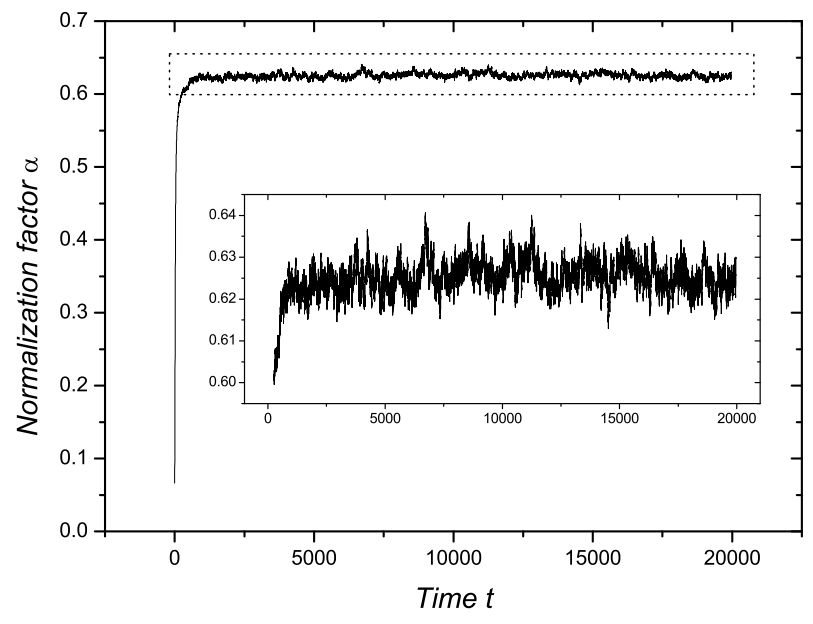

FIG. 1: Illustration of the normalization factor $\alpha$ as a function of time $t$ with the parameters $m=10$ and $M=30$. The amplified version can be seen in the inset. The data points correspond to system size $N=2 \times 10^{4}$, and each is obtained as an average of 100 independent runs.

where $\gamma=\frac{\alpha M}{m}+1$. Treating $k$ as continuous we write down the equation

$$
\frac{d p}{d k}=\frac{-\alpha-(\gamma-1) k}{k(k+\gamma-\alpha)} p(k),
$$

which yields the solution

$$
p(k) \sim k^{-\gamma+1} .
$$

When the system size $N$ is large compared with $M$, the degree distribution of the whole network $P(k)$ can be approximated by considering the inactive nodes only. Thus $P(k)$ can be calculated as the rate of the change of the degree distribution $p(k)$ of the active nodes. We find

$$
P(k)=-\frac{d p}{d k}=c k^{-\gamma}
$$

where $c=(\gamma-1) m^{\gamma-1}$ is the normalization constant. Finally, the exponent $\gamma=3$ is obtained from a selfconsistent condition

$$
2 m=\int_{m}^{\infty} k P(k) d k .
$$

The exponent $\gamma$ can be tunable if we introduce the initial attractiveness just like that of the model in Ref. [18]. Since it is not our focus, we will not show this effect here.

In Fig. 2, we plot the cumulative degree distribution of GKE networks by simulations. We obtain a power law scaling with best-fitted exponent $\gamma-1=1.96 \pm$ 0.02 , which is in agreement with the analytical result. In 


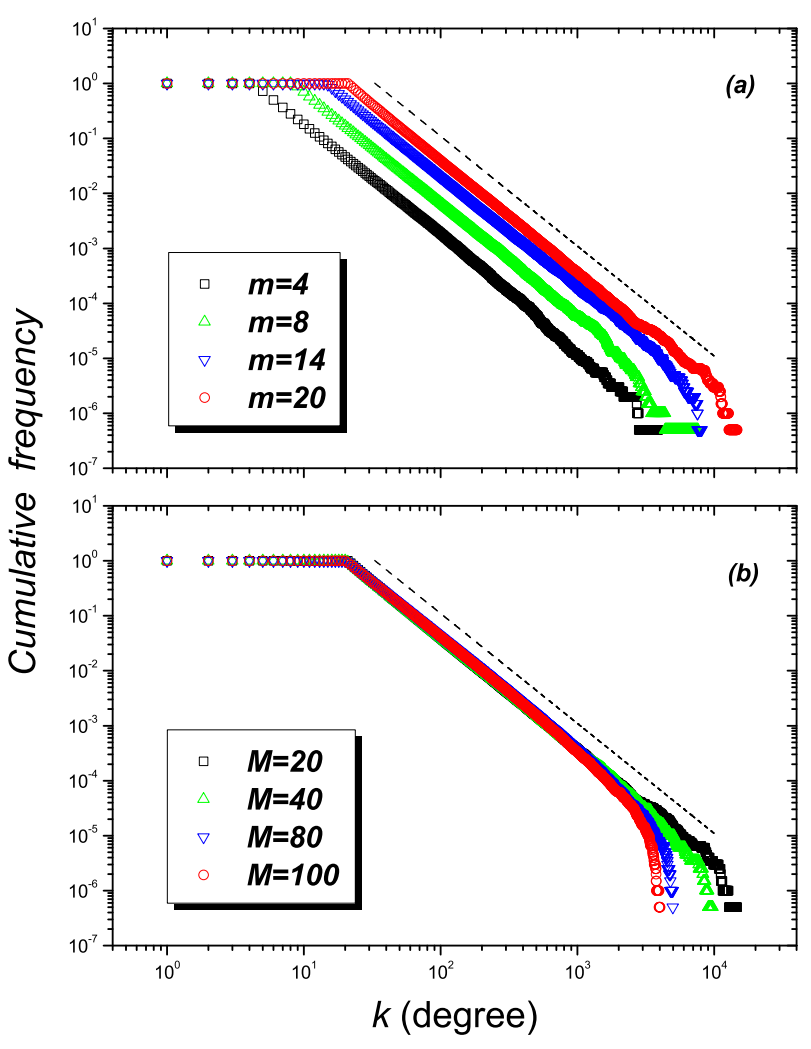

FIG. 2: (Color online) Cumulative degree distribution of GKE networks with parameters (a) $M=20 ; m=4$ (squares), 8(upward triangles), 14 (downward triangles), 20 (circles) and (b) $m=20 ; M=20$ (squares), 40(upward triangles), 80 (downward triangles), 100 (circles). The data points correspond to system size $N=2 \times 10^{4}$, and each is obtained as an average of 100 independent runs. The two dash lines have slope -2.0 for comparison.

fact, the exponent $\gamma$ is dependent on $m[20]$, which can be ignored when $m$ is large. However, the number of active nodes $M$ has no effect on degree distribution exponent $\gamma$, which is analytically and numerically obtained.

\section{B. Clustering coefficient}

The clustering coefficient $C(l)$ of node $l$ with degree $k_{l}$ can be defined as follow:

$$
C(l)=\frac{2 E(l)}{k_{l}\left(k_{l}-1\right)},
$$

where $E(l)$ is the number of links between neighbors of node $l$.

According to the definition of the GKE model, when a new node with $m$ links is added into the network, the links are attached to the nodes randomly selected from the active ones. Thus, the probability that two arbitrary active nodes are connected is $\frac{m}{M}$. It follows that a node $l$ with degree $k_{l}=m$ has

$$
E(l)=\frac{m}{M} \frac{k_{l}\left(k_{l}-1\right)}{2} .
$$

If $l$ is deactivated in the time step of its generation its neighborhood does not change any more and $C(l)$ keeps stable. Otherwise, node $l$ is not deactivated. In the next time step, a new node $j$ is added. As we note, the probability that node $j$ makes connection to $l$ is equal to the probability that one of the neighbors of node $l$ is deactivated in the last time step. We assume that if $k_{l}$ is added by 1 , one of its active neighbors has already been deactivated in the last time step. Thus, when the newly added node is connected to node $l$, one of its neighbors $s$ is inactive and one possible link between the newly added node and $s$ is missed. Then we have

$$
E(l)=\frac{m}{M}\left[\frac{k_{l}\left(k_{l}-1\right)}{2}-1\right],
$$

where $k_{l}=m+1$. Also, if $k_{l}=m+2$, there will be 2 inactive nodes in the neighbors of node $l$ causing another 2 possible links to be missed. Thus we obtain

$$
E(l)=\frac{m}{M}\left[\frac{k_{l}\left(k_{l}-1\right)}{2}-1-2\right],
$$

where $k_{l}=m+2$. This process repeats until node $l$ is deactivated, whose neighborhood does not change any more. By induction, we have

$$
E(l)=\frac{m}{M}\left[\frac{k_{l}\left(k_{l}-1\right)}{2}-\sum_{\nu=1}^{k_{l}-m} \nu\right]
$$

Thus the clustering coefficient $C(l)$ depends only on the degree $k_{l}$. The exact relation is

$$
C(l)=\frac{m}{M}\left[1-\frac{\left(k_{l}-m+1\right)\left(k_{l}-m\right)}{k_{l}\left(k_{l}-1\right)}\right] .
$$

The clustering coefficient $C$ of the whole network is the average of $C(l)$ over all nodes, i. e.,

$$
C=\frac{1}{N} \sum_{l=1}^{N} \frac{m}{M}\left[1-\frac{\left(k_{l}-m+1\right)\left(k_{l}-m\right)}{k_{l}\left(k_{l}-1\right)}\right] .
$$

Writing Eq. (14) in continuous form yields

$$
C=\int_{m}^{\infty} \frac{m}{M}\left[1-\frac{(k-m+1)(k-m)}{k(k-1)}\right] P(k) d k,
$$

where $P(k)$ is the degree distribution which we have derived above. Finally, the result is

$$
C=\frac{1}{M}\left[\frac{5 m}{6}-\frac{7}{30}+\mathcal{O}\left(m^{-1}\right)\right] .
$$

Obviously, when $M=m$, the clustering coefficient of the $\mathrm{KE}$ model is recovered[23]. 


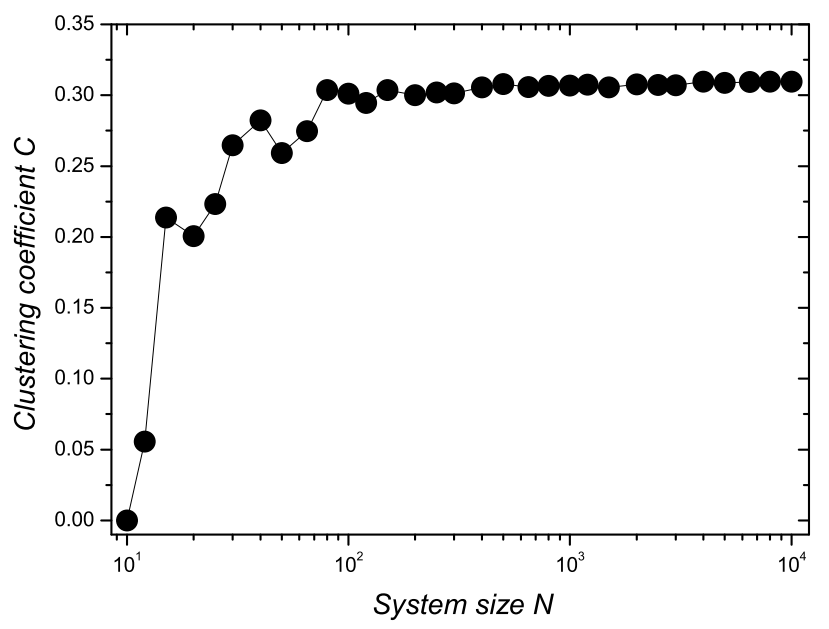

FIG. 3: Illustration of the average clustering coefficient $C$ as a function of system size $N$ with the parameter $m=4$ and $M=10$. The clustering coefficient $C$ approaches a stationary value about 0.31 , which is precisely predicted by Eq. (16). Each data point is obtained as an average of 1000 independent runs.

From Eq.(16), we know that the clustering coefficient $C$ is independent of the system size $N$. This asymptotic behavior of $C$ is reported in Fig. 3. In the limit of large $N$, the clustering coefficient $C$ gets to an stationary value of 0.31 , which agrees with the analytical result.

It is important to point out that the clustering coefficient has an novel scaling behavior $C \sim 1 / M$. Extensive numerical simulations perfectly confirm this result (see Fig. 4). This behavior can be related to the recent numerical study on the coauthor and paper citation networks, which will be discussed in Sec. V.

\section{STRUCTURAL PERTURBATION}

\section{A. Structural transition}

We introduce a structural perturbation to the GKE model by modifying step (1) of the definition as follow: Add a new node with $m$ links to the network. With probability $p$, attach one of the new node's links to a randomly selected inactive node. The other links are then attached to nodes chosen randomly from the $M$ active ones. We will show that the perturbation will lead to a phase transition[24] from large- to small-world in the network without changing the scale-free property.

In GKE model, each node can be represented by the time step of its generation. It is clear that, when $p=0$ the GKE network is structured[18], i. e., the time ordering exists and the mean field manner is absent[20,25]. We denote $l(t)$ as the average distance for pairs of nodes

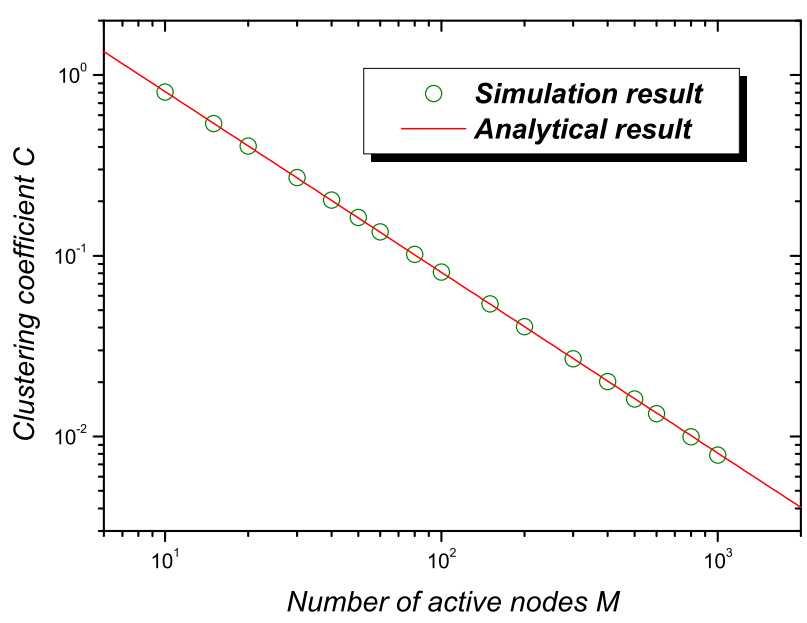

FIG. 4: (Color online) The analytical result of the clustering coefficient of GKE network, $C(M)$, as a function of $M$ (line), in comparison with the simulation (circles) results. Other parameters for the simulation are $m=10$ and $N=2 \times 10^{4}$. Each data point is obtained as an average of 100 independent runs.

separated by time interval $t$. Fig. 5 shows the simulation results of the variation of $l(t)$ with perturbation parameter $p$. It can be found that, when $p=0, l(t)$ increases linearly with $t$, i. e., the time ordering indeed exists. Since the nodes in the network are uniformly distributed on time axis, we can easily obtain that the average distance $L$ is linearly correlated to the system size $N$, i. e., $L \propto N$, which indicates the absence of small world effect. However, once $p$ is a small finite value, $l(t)$ becomes independent of time interval $t$, i. e., the time ordering vanishes. Meanwhile, all nodes with the same degree can be considered to be statistically equivalent, and the mean-field manner is recovered.

Let $d(i, j)$ denotes the distance between node $i$ and node $j$, and thus the average distance of the model with system size $N$ is

$$
L(N)=\frac{2 \sigma(N)}{N(N-1)}
$$

where the total distance is

$$
\sigma(N)=\sum_{1 \leq i<j \leq N} d(i, j)
$$

Intuitively, when a new node is added, the distance between old nodes will not increase. Hence we have

$$
\sigma(N+1) \leq \sigma(N)+\sum_{i=1}^{N} d(i, N+1),
$$




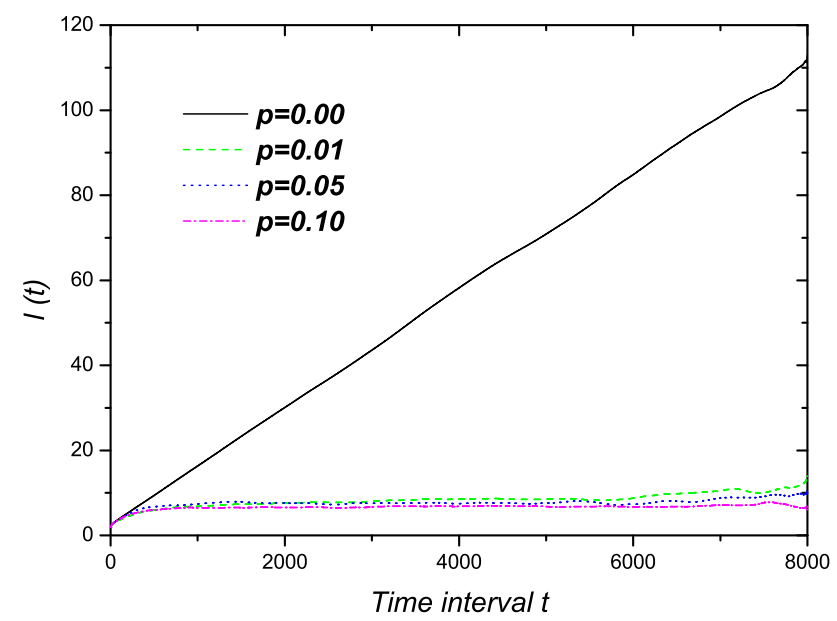

FIG. 5: (Color online) Illustration of $l(t)$ as a function of time interval $t$, with perturbation $p=0.00$ (solid line), 0.01 (dashed line), 0.05 (dotted line), and 0.10 (dot-dashed line). Other parameters for the simulations are $m=3, M=10$, and $N=8000$. Each data point is obtained as an average of 50 independent runs.

thus

$$
\sigma(N+1) \leq \sigma(N)+\sum_{i=1}^{N} d(i, x)+N
$$

where $x$ is the active node connected to the newly added one. Since $p$ is nonzero, by using mean-field approximation[26,27], we have

$$
\sum_{i=1}^{N} d(i, x) \approx L(N)(N-1) .
$$

Thus, the inequality (20) reduces to

$$
\sigma(N+1) \leq \sigma(N)+\frac{2 \sigma(N)}{N}+N .
$$

Rewriting (22) in continuous form will yield

$$
\frac{d \sigma(N)}{d N} \leq \frac{2 \sigma(N)}{N}+N
$$

which leads to

$$
\sigma(N) \leq N^{2} \ln N+B,
$$

where $B$ is a constant. As $\sigma(N) \sim N^{2} L(N)$ and $N$ is sufficiently large, we obtain $L(N) \leq \ln N$, i. e., the increasing tendency of $L(N)$ is not faster than $\ln N$, which predicts the presence of small-world property.

In fact, the GKE network is similar to a chain of dense clusters locally connected, i. e., it is like a regular lattice in topological view. For this peculiar topology, all

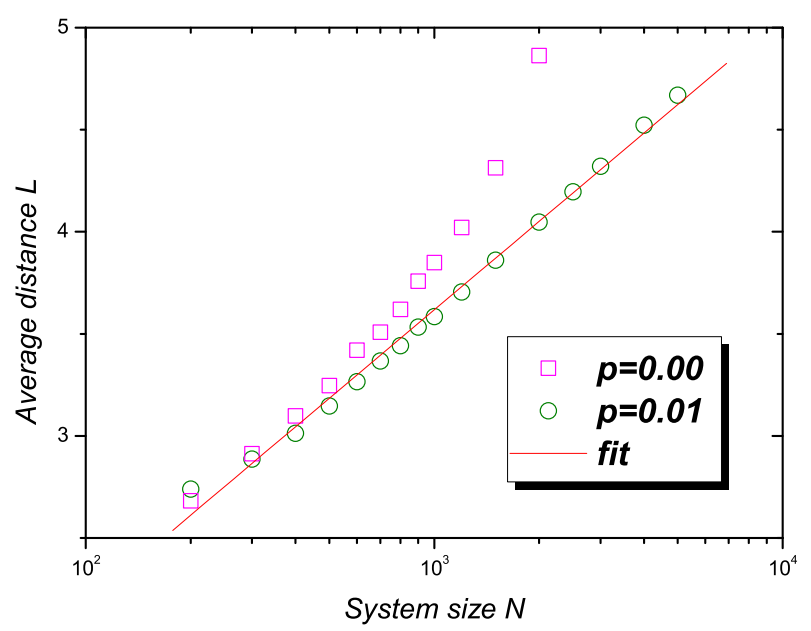

FIG. 6: (Color online) Illustration of the average distance $L$ as a function of $N$, with $p=0.00$ (squares) and $p=0.01$ (circles). When a perturbation $p=0.01$ is introduced, $L$ grows logarithmically with $N$. The values can be fitted well by a straight line, which is typical of the small world effect. Other parameters for these simulations are $m=4$ and $M=10$. Each data point is obtained as an average of 100 independent runs.

of the links in the network are local. When a perturbation is introduced, the network undergoes a cross-over from structured network to unstructured network. Actually, the perturbation just means that, with a probability, every node rewires one of its local links to a randomly selected node, which is precisely the definition of the model proposed by Watts and Strogatz[12]. That is to say, the cross-over is just the small-world phase transition[24].

In Fig. 6, we show the dependence of average distance $L$ on system size $N$ with $p=0.00$ and $p=0.01$ in GKE network. For $p=0.00$, the average distance grows linearly $L \propto N$, the same behavior observed in one-dimensional regular lattices. Once $p$ is a small finite value, $L$ becomes logarithmic related to $N$, i. e., $L \propto \ln N$. The logarithmic increase of average distance with system size predicts that the phase transition from large- to small-world occurs, which is in agreement with the analytical result.

It should be noted that, although we introduce a structural perturbation into the network, the scale-free property is not affected and the power-law exponent $\gamma=3$ is maintained. Numerical simulations shown in Fig. 7 confirm this feature. 


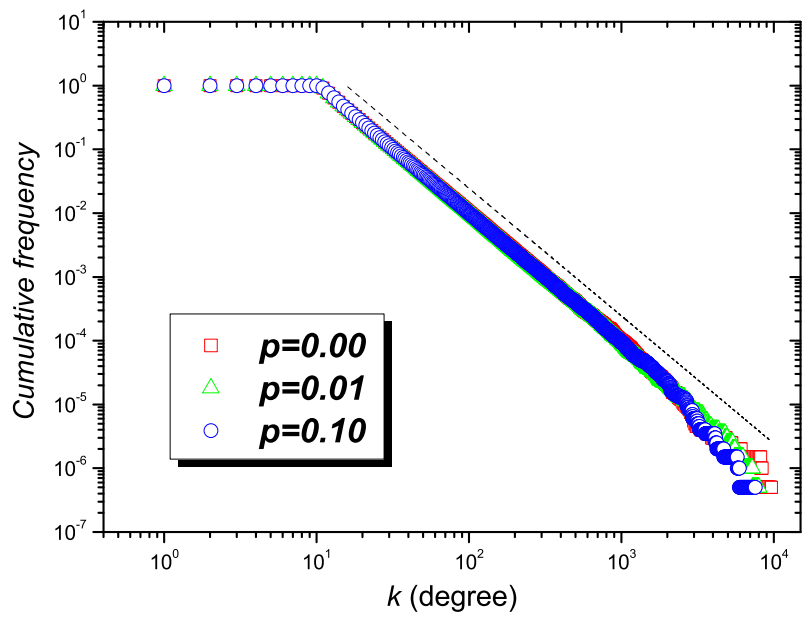

FIG. 7: (Color online) Illustration of the cumulative degree distribution of the GKE network with perturbation $p=0.00$ (squares), 0.01 (upward triangles), and 0.10 (circles). The fitted power-law exponent is $\gamma-1=1.97 \pm 0.02$. Other parameters for these simulations are $m=10, M=20$, and $N=2 \times 10^{4}$. Each data point is obtained as an average of 100 independent runs. The dashed line has slope -2.0 for comparison.

\section{B. Universal scaling behavior of clustering coefficient}

In the following subsection, we investigate the dependence of clustering coefficient $C$ on perturbation parameter $p$. Analogous to the derivation of clustering coefficient in GKE network without perturbation, we give an approximately analytical result. According to the modification of the model, when a new node $l$ with $m$ links is added into the network, one of the links is attached to a randomly selected inactive node $s$ with probability $p$. That is to say, with probability $p$, one of the neighbors of $l$ is inactive. Since the system size $N$ is large compared with $M$, we assume that node $s$ is apart from the active nodes[28]. Thus, $m-1$ possible links between neighbors of $l$ are missed. Furthermore, node $s$ is always apart from the afterward added nodes that are connected to node $l$, which causes another $k-m$ possible links missed. Thus we have

$$
\begin{aligned}
C(l) & =\frac{m-1 \times p}{M}\left[1-\frac{\left(k_{l}-m+1\right)\left(k_{l}-m\right)}{k_{l}\left(k_{l}-1\right)}\right] \\
& -p \frac{m-1 \times p}{M} \frac{2(m-1)}{k_{l}\left(k_{l}-1\right)} \\
& -p \frac{m-1 \times p}{M} \frac{2\left(k_{l}-m\right)}{k_{l}\left(k_{l}-1\right)} .
\end{aligned}
$$

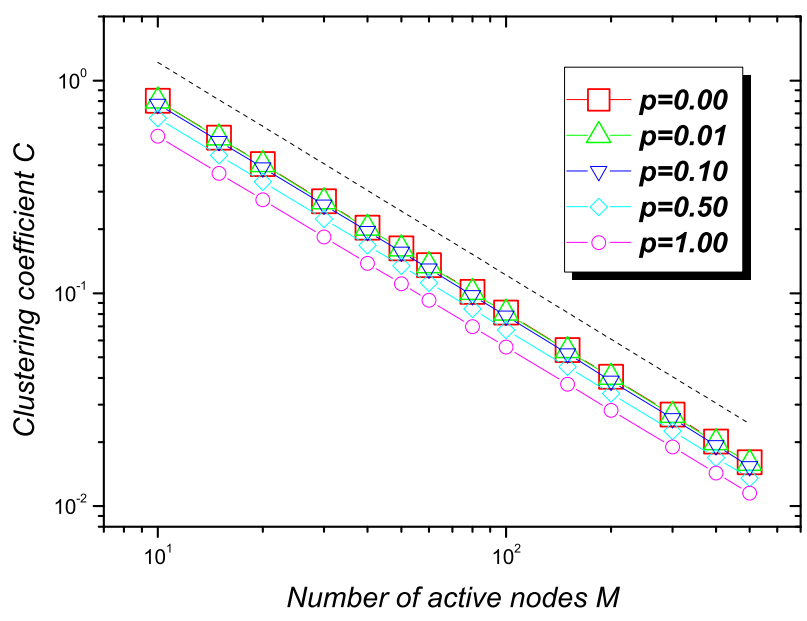

FIG. 8: (Color online) Illustration of the clustering coefficient $C$ as a function of number of active nodes $M$, with perturbation parameter $p=0.00$ (squares), $p=0.01$ (upward triangles), $p=0.10$ (downward triangles), $p=0.50$ (diamonds), and $p=1.00$ (circles). The average fit slope for the simulations is 0.994. Other parameters for these simulations are $m=10$ and $N=2 \times 10^{4}$. Each data point is obtained as an average of 100 independent runs. The dashed line has slope -1.0 for comparison.

Similar to the derivation of Eq. (16), we have

$$
C=\frac{m}{M}\left(\frac{5}{6}-\frac{7}{30 m}\right)-\frac{1}{M}\left(\frac{13}{6}-\frac{7}{30 m}\right) p+\mathcal{O}\left(p^{2}\right)
$$

It is worthwhile to note that the scaling behavior $C \sim 1 / M$ is conserved though there exist certain fluctuations in the network which lead to a structure transition. That is to say, $C \sim 1 / M$ is a universal scaling behavior of clustering coefficient induced by deactivation mechanism. Fig. 8 shows the log-log plot of the clustering coefficient $C$ versus $M$ with different perturbation parameters obtained by simulations. We can see that the perturbation has almost no effect on the scaling behavior of $C$, which agrees well with the analytical result.

From Eq. (26), we know that, when $p$ is sufficiently small, the clustering coefficient $C$ has a linearly relation with $p$. Fig. 9 shows the simulation result of clustering coefficient $C$ as a function of perturbation parameter $p$, with $m=4$ and $M=10$. The slope found numerically is 0.223 , slightly larger than the analytical result $\frac{1}{M}\left(\frac{13}{6}-\frac{7}{30 m}\right)=0.21$. The deviation is due to the approximation[28] used in the theoretical derivation of $C$. It is clear that the node $s$ is not always apart from all the active nodes, which actually causes less than $m-1$ possible links missed between neighbors of $l$. Thus, we can easily find the precise slope should be a little larger than that obtained from Eq. (26). 


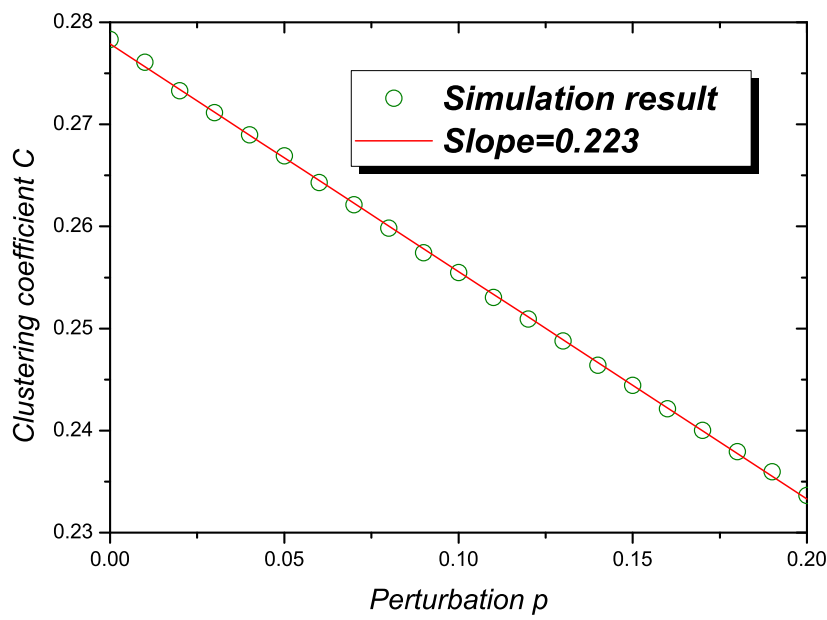

FIG. 9: (Color online) Illustration of the clustering coefficient $C$ as a function of perturbation parameter $p$. The fit slope is 0.223 . Other parameters for this simulation are $m=4$, $M=10$, and $N=2 \times 10^{4}$. Each data point is obtained as an average of 100 independent runs.

\section{DISCUSSIONS AND CONCLUSIONS}

We first discuss the relationship between the numerical behavior of clustering coefficient in the coauthor and paper citation networks and our theoretical result. According to the model of Ref. [19], each of the authors and papers is assigned a topic, and authors can only cite, produce papers or coauthor with others in their own topic area. It means that, by topics the whole network is divided into many subnetworks which evolve separately and simultaneously. Each of the subnetworks can be reduced to a GKE network and the number of these GKE subnetworks is just the number of the topics denoted as $n$. In each subnetwork, the number of active authors who are doing research or the number of active papers that are likely to be cited just corresponds to the number of active nodes in GKE network, which is denoted as $M$. Since the whole network is divided into $n$ subnetworks, we intuitively know that the number of active authors or papers in each subnetwork is inversely proportional to the number of topics, i. e., $M \sim 1 / n$. For each subnetwork can be treated as a GKE network, incorporating with our theoretical result $C \sim 1 / M$, we can easily obtained that $C \sim n$. Since each subnetwork evolves parallelly, the clustering coefficient of the whole network has the same behavior that it is linearly correlated with the number of topics. Therefore, by using our theoretical result we can indicate that the numerical behavior of clustering coefficient in the coauthor and paper citation network presented in Ref. [19] is due to the deactivation mechanism. Furthermore, in the above discussion, we reduce the aging mechanism to deactivation mechanism. In fact, in the model of Ref. [19], the aging effect is introduced by an aging function. To this point, we conjecture that there might be similar scaling behaviors of $C$ in networks generated by other forms of aging mechanism.

Finally, it is worthwhile to point out that, to our knowledge, no empirical data are available to illustrate the theoretical scaling behavior of clustering coefficient. Nevertheless, this interesting property is due to the deactivation process which is a special case of aging effect. In network evolution, aging is a universal mechanism. Therefore, this simple theoretical result of $C$ will have a rich practical significance and potential applications in future network research. Meanwhile, such scaling behavior of $C$ should be given further considerations from empirical investigations.

In summary, motivated by the aging effect governing the evolution of the coauthor and paper citation networks, a generalized deactivation model of network called GKE is presented in this paper. We study analytically and by simulations several topological features of this model, such as the degree distribution and clustering coefficient. Most importantly, an interesting scaling behavior of the clustering coefficient $C \sim 1 / M$ is obtained, which shows that the numerical result recently observed in the coauthor and paper citation networks is due to deactivation mechanism. By introducing a perturbation, the GKE network undergoes a small-world phase transition, while the scaling behavior of $C$ is conserved. It indicates that $C \sim 1 / M$ is a universal scaling behavior of clustering coefficient induced by deactivation mechanism. In addition, we would like to emphasize that our study unifies the concept of regular lattice, small-world graphs and scale-free networks in a single model, and the GKE model generalizes the new class of the networks with a crucial parameter $M$.

Since the GKE networks present peculiar structure property, it will be interesting to investigate the effect of their complex topology features on the network dynamics[25,29-31]. Especially, the clustering coefficient of GKE network is precisely tunable by parameter $M$ or $p$ without changing the degree distribution. Therefore, the model can be used to quantitatively study the effect of clustering on network synchronization[32-34] and network epidemics[31,35]. Research along this line is in progress.

\section{ACKNOWLEDGMENTS}

Shi thanks the program for New Century Excellent Talents in University of China for financial support (NECT04-0510). This work is also supported by National Natural Science Foundation of China under Grant Nos. 70471084 and 10372045 . 
[1] R. Albert and A.-L. Barabási, Rev. Mod. Phys. 74, 47 (2002).

[2] S. N. Dorogrovtsev and J. F. F. Mendels, Adv. Phys. 51, 1079 (2002).

[3] M. E. J. Newman, SIAM Rev. 45, 167 (2003).

[4] M. Faloutsos, P. Faloutsos and C. Faloutsos, Computer Communications Review 29, 251 (1999).

[5] R. Pastor-Satorras, A. Vazquez, and A. Vespignani, Phys. Rev. Lett. 87, 258701 (2001)

[6] B. A. Huberman, The Laws of the web (MIT Press, Cambridge, 2001).

[7] R. Albert, H. Jeong and A. -L. Barabási, Nature 401, 130 (1999).

[8] H. Jeong, S. P. Mason, A.-L. Barabási, and Z. N. Oltvai, Nature 411, 41 (2001).

[9] E. Ravasz, A. L. Somera, D. A. Mongru, Z. N. Oltvai, and A.-L. Barabási, Science 297, 1551 (2002).

[10] S. L. Pimm, Food Webs (University of Chicago Press, Chicago, 2002).

[11] S. Wasserman and K. Faust, Social Network Analysis (Cambridge University Press, Cambridge, 1994); J. Scott, Socical Network Analysis: A Handbook (Sage Publications, London, 2000).

[12] D. J. Watts and S. H. Strogatz, Nature 393, 440 (1998).

[13] A. -L. Barabási and R. Albert, Science 286, 509 (1999).

[14] L. A. N. Amaral, A. Scala, M. Barthélémy, and H. E. Stanley, Proc. Natl. Acad. Sci. U.S.A. 97, 11149 (2000).

[15] S. N. Dorogovtsev and J. F. F. Mendes, Phys. Rev. E 62, 1842 (2000).

[16] A. F. J. Van Raan, Scientometrics 47, 347 (2000);

[17] H. Zhu, X. Wang, and J.-Y. Zhu, Phys. Rev. E 68, 056121 (2003); K. B. Hajra and P. Sen, Phys. Rev. E 70, 056103 (2004); K. B. Hajra and P. Sen, Physica A 346, 44 (2005); P.-Q. Jiang, B.-H. Wang, T. Zhou, Y.-D. Jin, Z.-Q. Fu, P.-L. Zhou, and X.-S. Luo, Chin. Phys. Lett. 22, 1285 (2005).

[18] K. Klemm and V. M. Eguíluz, Phys. Rev. E 65, 036123 (2002).

[19] K. Börner, J. T. Maru, and R. L. Goldstone, Proc. Natl.
Acad. Sci. U.S.A. 101, 5266 (2004).

[20] A. Vázquez, M. Boguñá, Y. Moreno, R. Pastor-Satorras, and A. Vespignani, Phys. Rev. E 67, 046111 (2003).

[21] C.-P. Zhu, S.-J. Xiong, Y.-J. Tian, N. Li, and K.-S. Jiang, Phys. Rev. Lett. 92, 218702 (2004).

[22] It means that each node is connected symmetrically to its $2 z$ nearest neighbors. For detailed definition, see B. Bollobás, Random Graphs (Academic Press, New York, 1985). We set $z=1$ in the simulations in this paper for the sake that the influence of initial graph on the evolving of the network is minimal and can be ignored.

[23] K. Klemm and V. M. Eguíluz, Phys. Rev. E 65, 057102 (2002).

[24] M. E. J. Newman and D. J. Watts, Phys. Rev. E 60, 7332(1999).

[25] V. M. Eguíluz, E. Hernández-García, O. Piro, and K. Klemm, Phys. Rev. E 68, 055102(R) (2003).

[26] T. Zhou, G. Yan, and B.-H. Wang, Phys. Rev. E 71, 046141 (2005).

[27] Z.-M. Gu, T. Zhou, B.-H. Wang, G. Yan, C.-P. Zhu, and Z.-Q. Fu, arXiv: cond-mat/0505175

[28] As we know, when $p=0$, the topology of the GKE network is similar to a one-dimensional lattice. Thus, if $p$ is sufficient small, this approximation is reasonable.

[29] K. Klemm, V. M. Eguiluz, R. Toral, and M. SanMiguel, Phys. Rev. E 67, 026120 (2003)

[30] K. Suchecki, V. M. Eguíluz, and M. S. Miguel, Phys. Rev. E 72, 036132 (2005)

[31] V. M. Eguíluz and K. Klemm, Phys. Rev. Lett. 89, 108701 (2002)

[32] P. N. McGraw, M. Menzinger, Phys. Rev. E 72, 015101(R) (2005).

[33] M. Zhao, T. Zhou, B. -H. Wang, G. Yan, and H. -J. Yang, preprint arXiv: cond-mat/0510332

[34] X. Wu, B. -H. Wang, T. Zhou, W. -X. Wang, M. Zhao, and H. -J. Yang, Chin. Phys. Lett. 23, 1046 (2006).

[35] M. Boguñá, R. Pastor-Satorras, and A. Vespignani, Phys. Rev. Lett. 90, 028701 (2003). 